\title{
A NOVEL CROWD MANAGEMENT SYSTEM TO MAINTAIN PHYSICAL DISTANCING IN PUBLIC TRANSPORT TO STOP THE SPREAD OF COVID-19 VIRUS
}

\author{
Arkajyoti Mitra ${ }^{1}$, Joyanto Roychoudhary ${ }^{2}$ \\ ${ }^{1}$ Student, Electronics and Communication Engineering, Meghnad Saha Institute Of Technology, \\ Kolkata, West Bengal, India. \\ ${ }^{2}$ Faculty Advisor, Assistant Professor in the Department Of Electronics and Communication \\ Engineering, Meghnad Saha Institute Of Technology, Kolkata, West Bengal, India.
}

\begin{abstract}
With the rising cases of COVID-19 worldwide, transportation sector has been one of the primary victims of Covid-19. Our country is going through the unlocking phase and public transport is being available again. But in order to stay safe from Covid-19, the physical distancing norms must be maintained. In public transport, it is rather impossible to maintain a proper physical distance. So we have created an automatic system, where the number of people riding in the vehicle is always fixed so as to maintain proper physical distancing. This system has a fixed number of passengers who can ride at the same time. It detects the no. of passengers going inside the vehicle and stops the entry of other passengers when it reaches it's permissible range. And until the passenger count is lessened, the entry gate won't open. The Novel prototype model is tested using web-based simulation tool Tinker Cad Circuits and the outcomes are remarkable and can be Implemented in Real life Cases for crowd management systems.
\end{abstract}

Keywords: Transportation, Unlocking, Physical Distancing, Permissible, Prototype.

\section{INTRODUCTION}

World Health Organization (WHO) has instructed to maintain social distancing to prevent the spread of Covid-19 virus. Physical distancing of at least 1 metre between each other in both outdoor and indoor should be ensured to stay safe from the virus. With the upliftment of lockdown rules in different countries, the people are trying to get back to their normal lifestyle. And in doing so, many of them are violating the covid protocols that should be maintained while travelling in public transport. Most of the time, the public vehicle is overcrowded, thus risking their lives to Covid. The only way to control this situation is to monitor the no. of people accommodating into a public transport at a time. The public transport should have automated doors which should be touch free. The automated doors works on the principle of actuating the servo on entering through a door whenever the sensor observes a low distance reading due to an obstruction at a line-of-sight distance from the sensor. When a person comes near the door, obstruction in the line-of-sight of the sensor takes place, soon the Arduino board receives a low distance reading and instructs the servo motor to actuate and open the door of the vehicle. When the vehicle reaches its maximum occupancy (pre-determined) the door closes and is not opened for entry until someone leaves the vehicle, thereby decreasing the number of people in the vehicle and making room for new incoming passengers. The number of people actually entering and exiting the vehicle can be counted using various types of sensors and the most popular sensor is an ultrasonic sensor which works on the principle of a RADAR. They are mainly used to determine the distance of the human from the entry gate. As the person is in the vicinity of $10-20 \mathrm{~cm}$, the system gets activated and it increases the counter value by 1 and thus the LED display attached to it shows the no. of persons entering and exiting the vehicle.

\section{PROPOSED METHODOLOGY}

The ultrasonic sensor functions on the principle of the SONAR (Sound Navigation and Ranging) and RADAR (Radio Detection and Ranging) system. Ultrasonic sensors are mainly used to determine the distance of the human from the entry gate. As the person is in the vicinity of $10-20 \mathrm{~cm}$, the system gets activated and it increases the counter value by 1 . This counter value is then displayed in the LCD monitor as the total number of persons inside. It also welcomes the incoming passenger with a 
"WELCOME" message in the LCD display. A similar ultrasonic sensor is attached on the exit gate. As the person is in the vicinity of $10-20 \mathrm{~cm}$ of the exit gate, it activates the machine and decreases the counter value by 1 , which is then displays a "BYE" message to the person leaving the vehicle entirely. Both the sensors work mutually together to maintain a fixed number of people inside the vehicle. If the maximum limit is reached, the system with the help of an actuating servo motor closes the door, until someone leaves the vehicle, thereby decreasing the number of people inside the vehicle and making room for new incoming passengers to enter. All of this process works continuously to provide a seamless and safe travelling experience.

\section{THE PROPOSED MODEL}

The proposed model is simulated using web-based simulation tool Tinker Cad Circuits. Tinker cad Circuits is an online platform that allows users to virtually connect the components/sensors via code and realize the practical circuit online without the requirement of any hardware components. The Figure below shows the circuit arrangement for the Covid-19 Crowd Management Machine for public transport.

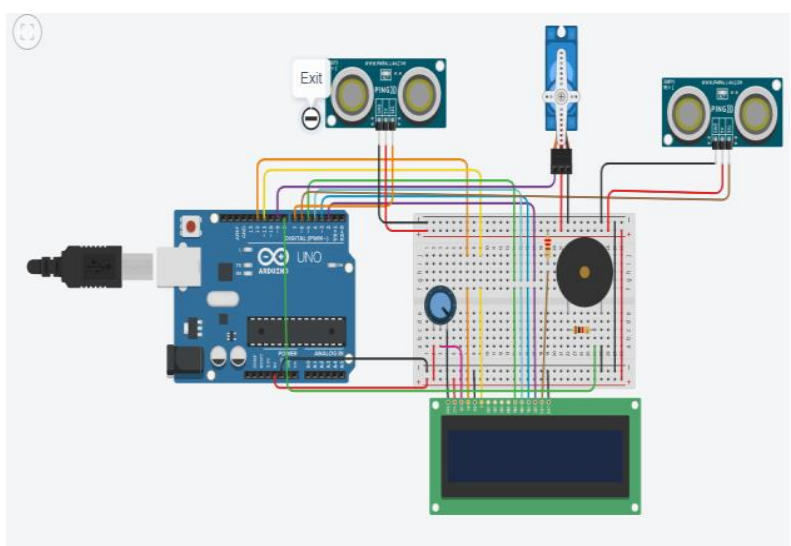

Fig.1.The Proposed Model

\section{RESULTS \& DISCUSSION}

The simulated outcomes achieved were the same as expected.

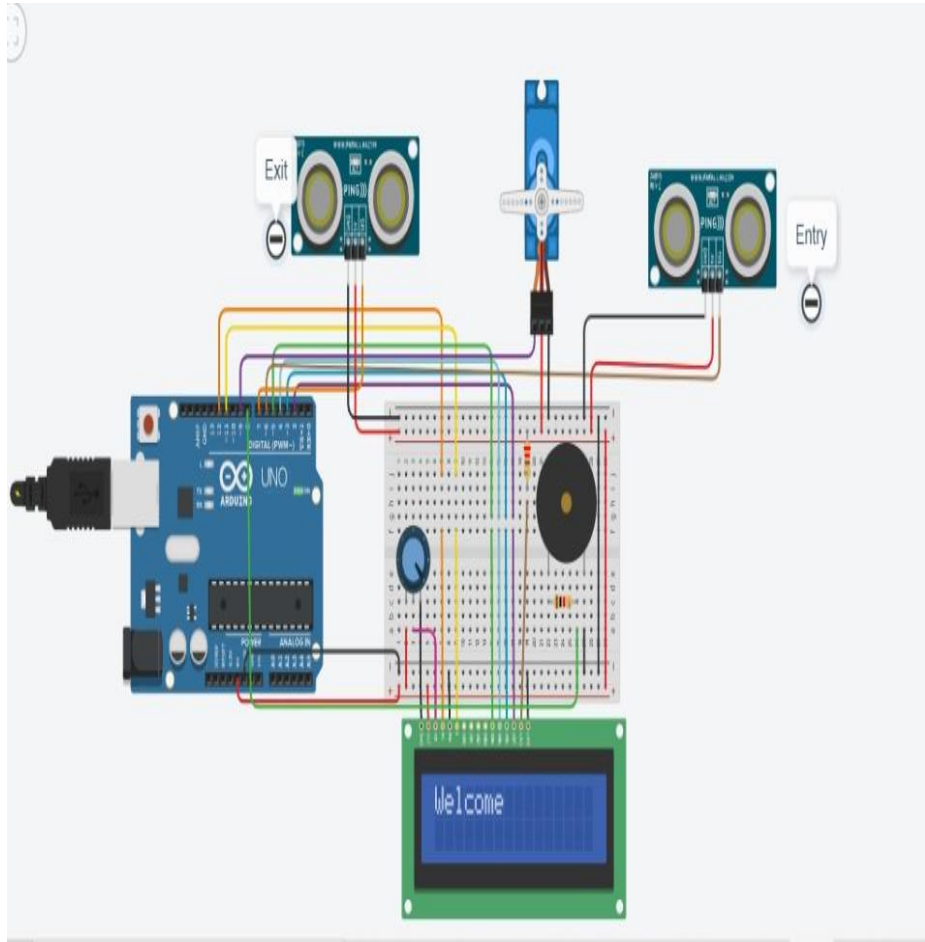

Fig.2. Welcome

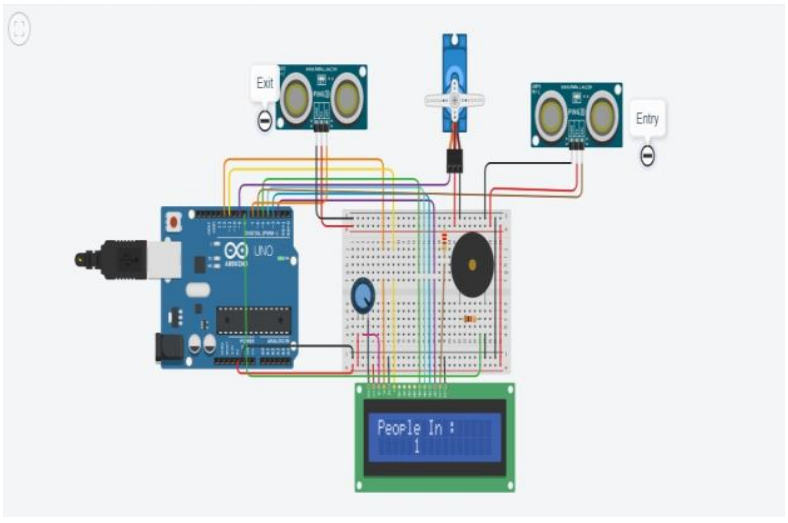

Fig.3. People inside 1

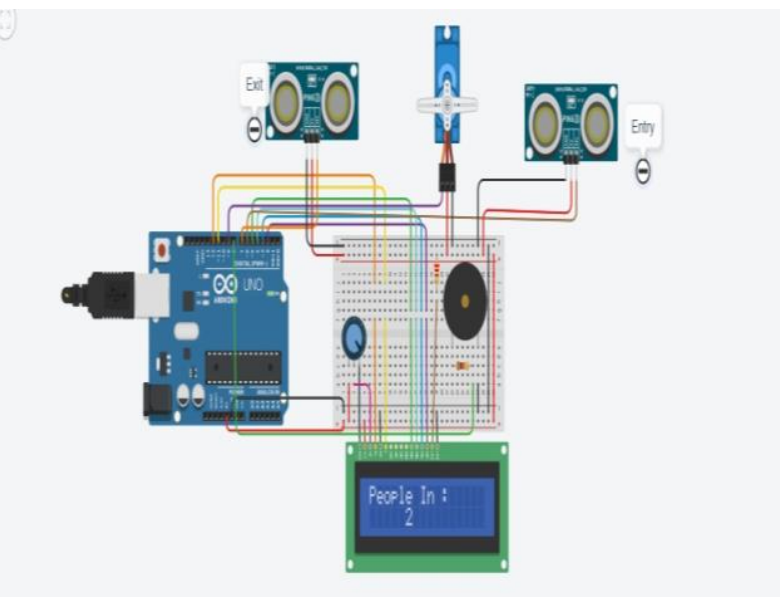

Fig.4. People inside 2 


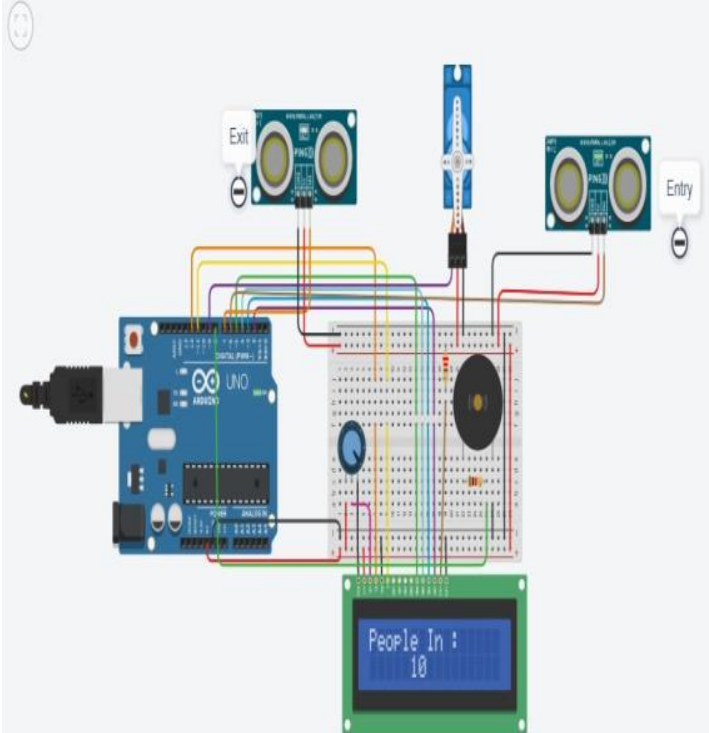

Fig.5. People inside 10 (Max limit)

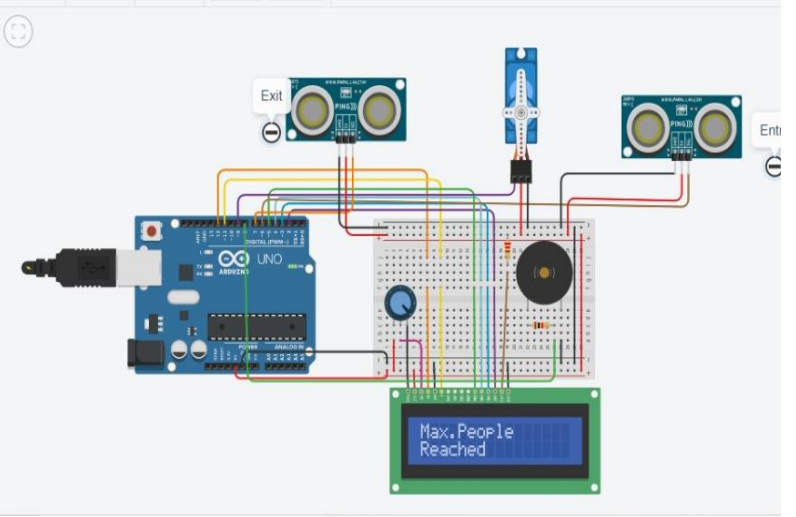

Fig.6. Maximum People Inside.

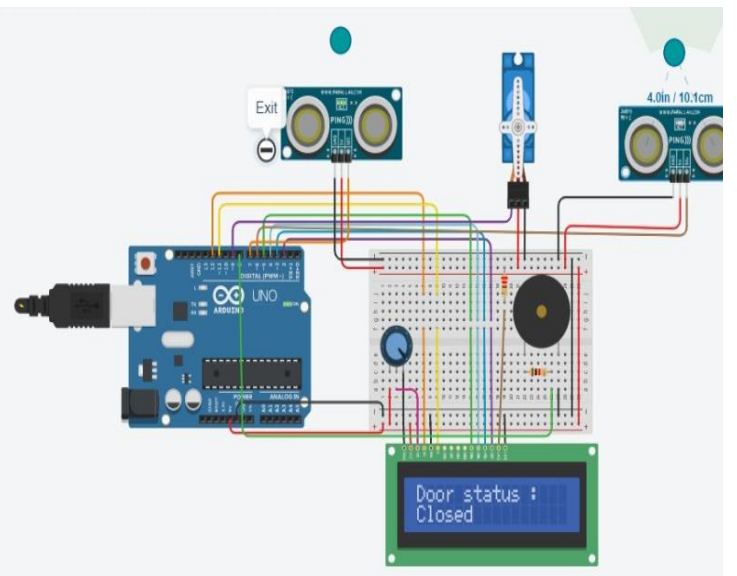

Fig.7. Door Status Closed.

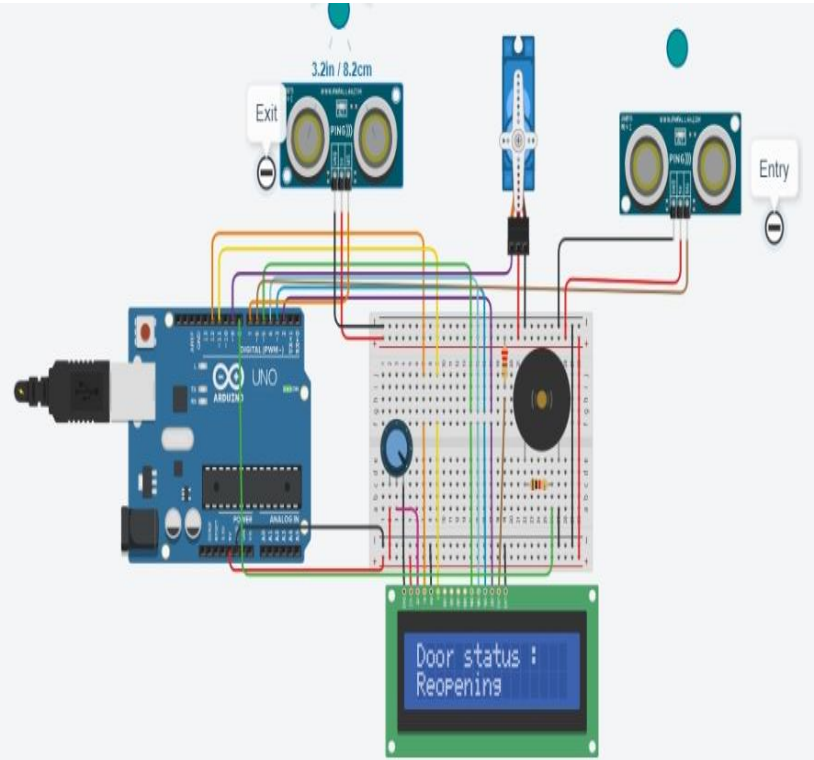

Fig.8. Door Status Reopening.

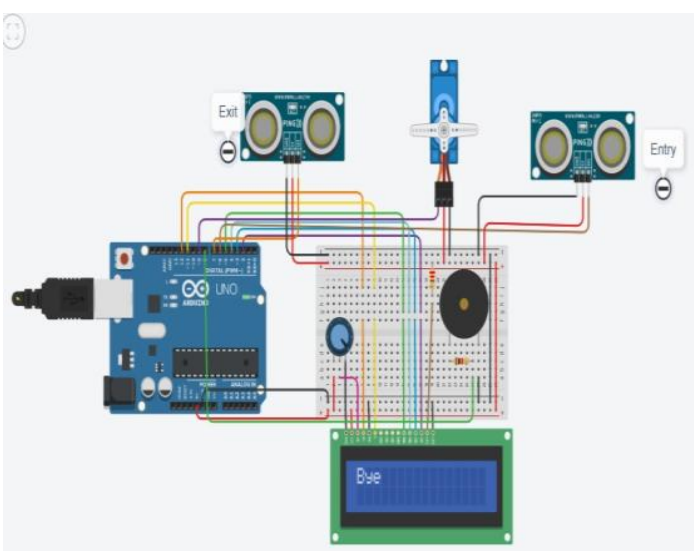

Fig.9. Bye (When people start leaving)

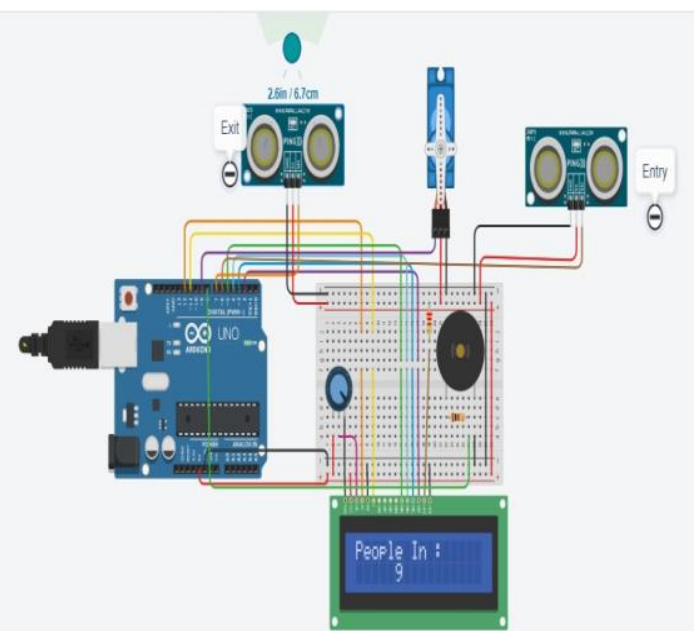

Fig.10. No. of people inside after they start leaving. 


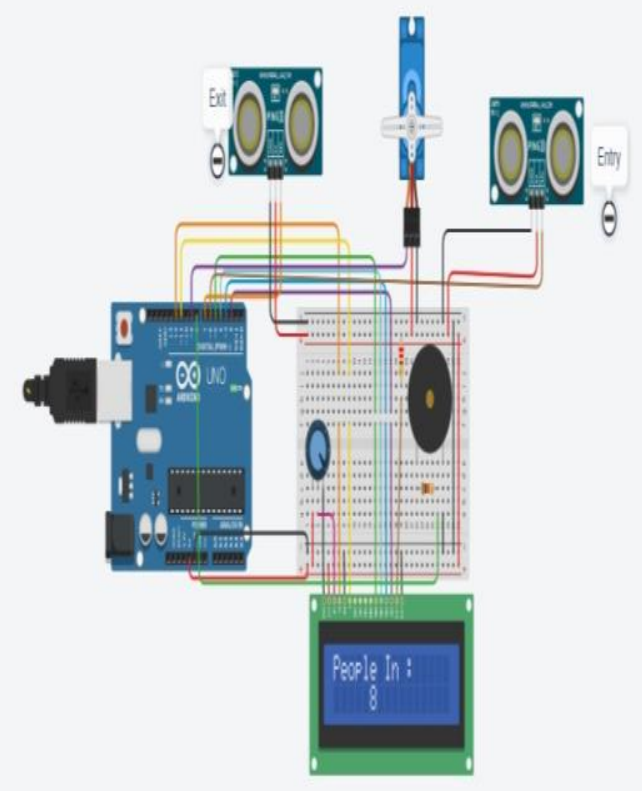

Fig.11. No. of people inside after they continue to leave.

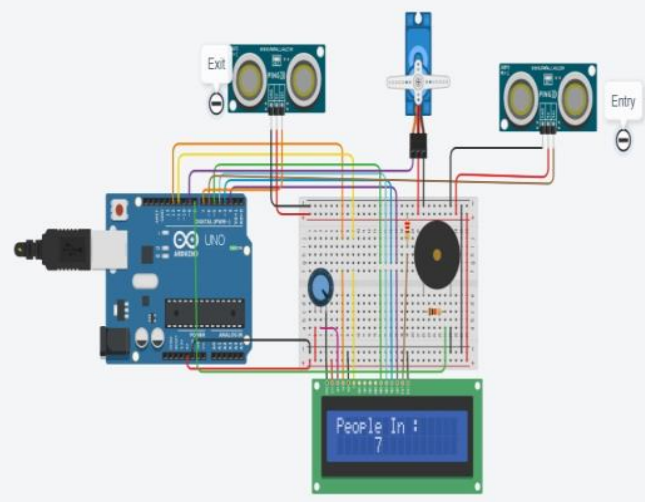

Fig.12. No. of people inside after they continue to leave.

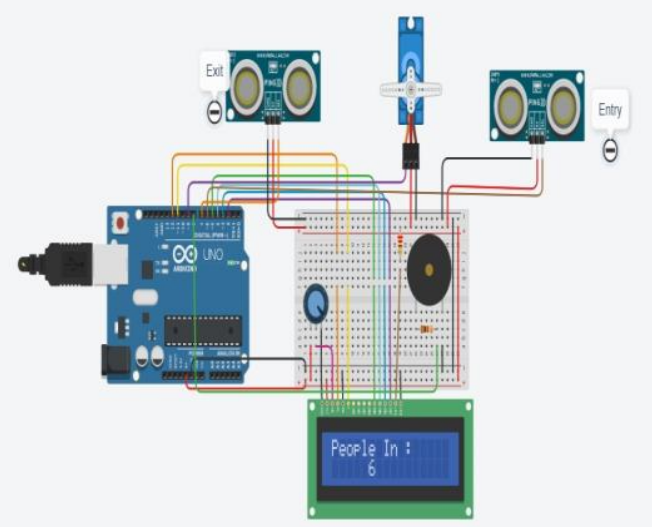

Fig.13. No. of people inside after they continue to leave.

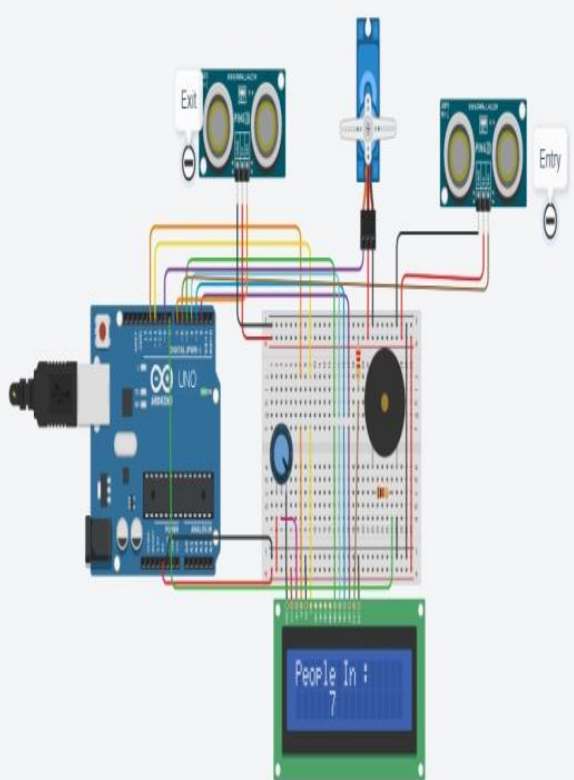

Fig.14. No. of people when they start entering again

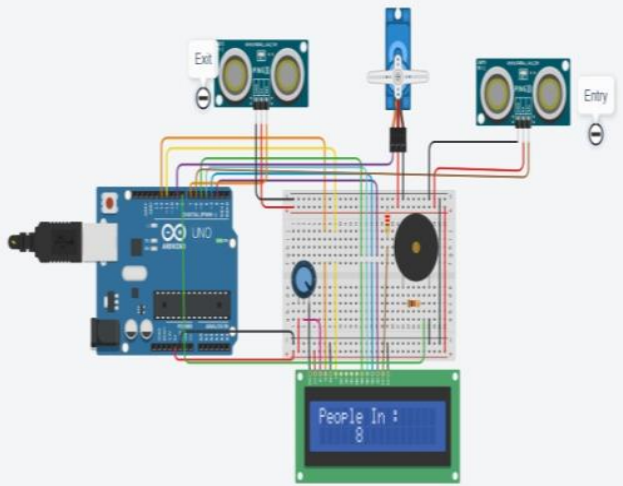

Fig.15. No. of people when they start entering again

Table1.Crowd Management System Monitoring and Result

\begin{tabular}{|llr|}
\hline CONDITION & RESULT \\
\hline $\begin{array}{l}\text { When max. people } \\
\text { inside }\end{array}$ & $\begin{array}{l}\text { Door } \\
\text { automatically, roses } \\
\text { turns on, Alert message } \\
\text { displayed, } \\
\text { prohibited. }\end{array}$ \\
\hline $\begin{array}{l}\text { When people less than } \\
\text { maximum inside }\end{array}$ & $\begin{array}{l}\text { Door } \\
\text { automatically. Buzzer } \\
\text { turned off, Entry } \\
\text { accessed. }\end{array}$ \\
\hline
\end{tabular}




\section{NOVELTY \& CONTRIBUTION}

Things Speak is an IoT Analytics platform that allows users to aggregate, visualize and analyze live data streams in a cloud. In our prototype we can send data to Thing speak cloud for real time monitoring of the number of the person allowed inside. Data analysis can be used to monitor the number of passengers riding the public transport on a daily basis and the obtained data might help in increasing the number of available vehicles for the public use in order to strictly abide by the Covid- 19 norms. GPS (Global Positioning System) can be used within the vehicle perimeters, to track the passengers and their seating positions. It should ensure that a proper physical distancing should be maintained between the passengers inside so as to prevent the spread of Covid-19 virus. IOT node consists of a mobile in every passengers hand with COVID-19 tracker app along with it a BLE Radar Dashboard for social distancing in the app to monitor the overcrowdings inside the vehicle when all people are in. Also we are using GSM module as it can send notification to individual passenger for the maintenance of social distancing norms inside the vehicle. This work can also be implemented in real life situation due to its remarkability and robustness. The novelty could not be implemented due to the reason that loading sensory data to cloud needs ESP8266 Wi-Fi module which is disabled in Tinker Cad software due to security reasons.

\section{CONCLUSION}

The proposed system is the design of an automatic Covid-19 Crowd Management Machine using Arduino technology that screens a human from a distance of 10-20 cm of the machine and lets the person to enter/exit from the vehicle. The machine keeps count of the number of people inside and prohibits from entering the vehicle once the maximum limit is reached, thus helping to prevent the vehicle from being overcrowded. The design implementation takes into consideration the covid19 pandemic situation. The experimental results have been conducted and analyzed using an online circuit Simulator Tinker Cad Circuits. From the overall analysis, we can conclude that such an automatic, efficient crowd management machine for public transport can be built at a low price. In future more efficient Automated Crowd Management Machine can be developed using AI Technology.

\section{REFERENCES}

[1.] Internet of things (IoT) applications to fight against COVID-19 pandemic Ravi Pratap Singh, Mohd Javaid, Abid Haleem, Rajiv Suman Diabetes Metab Syndrome. 2020 July-August; 14(4): 521-
524. Published online 2020 May 5. doi: 10.1016/j.dsx.2020.04.041.

[2].J. Blake and S. Ohi, "The development of LCD FT displays", Electronic Product Design, vol. 22, pp. 30-33, Dec. 2001.

[3]. H. A. Abdulqader, B. G. Varghese and N. A. Nabhani, "Dynamic short distance estimation using ultrasonic," 2012 IEEE Business, Engineering \& Industrial Applications Colloquium (BEIAC), 2012, pp. 70-73, doi: 10.1109/BEIAC.2012.6226109.

[4]. M Sveda, P. Benes, R. Vrba and F. Zezulka, "Introduction to Industrial Sensor Networking," A book. chapter in: M. Ilyas and I. Mahgoub, (Eds.), Handbook of Sensor Networks: Compact Wireless and Wired Sensing Systems, CRC Press LLC, Boca Raton, FL, USA, 2004.

[5]. Haleem A., Javaid M., Khan I.H. Internet of things (IoT) applicationsinorthopaedics. J. Clin. Orthop.Trauma. 2019 doi: 10.1016/j.jcot.2019.07.003. In press. 